$\xi$

\title{
Detection of Microaneurysms and Hemorrhages in Fundus Image for Glaucoma Diagnosis
}

\author{
R. Shanthi ${ }^{1^{*}}$, S. Prabakaran ${ }^{2}$ \\ ${ }^{1}$ Research scholar, Department of CSE, SRM Institute of Science \& Technology, Chennai-603 203, Tamil Nadu, India \\ ${ }^{I}$ Assistant Professor, Valliammai Engineering College, Chennai 603 203, Tamil Nadu, India \\ ${ }^{2}$ Professor, Department of CSE, SRM Institute of Science \& Technology, Chennai 603 203, Tamil Nadu, India \\ *Corresponding Author Email: shanthirajaji@gmail.com
}

\begin{abstract}
Eye is the most sensitive and valuable organ of vision which helps us to visualize the world around us. Due to the high pressure in the eye, the optic nerve fails to transmit the signal to the brain; such a disorder is called Glaucoma. Detection of Microaneurysms and Hemorrhages are validated from the fundus image. To extract the shape features Morphological image flooding is used. In this approach, candidate regions are first segmented, Feature Extraction is done by Dynamic Shape Features. Further the classification process is carried out using Random Forest (RF) classifier method. The process of screening is evaluated on publicly available database Diaretdb1 with the resolution of $1152 \mathrm{x} 1500$ pixels for both healthy and abnormal images. The performance of segmentation in this method is evaluated in terms of sensitivity, specificity, and segmentation accuracy. In future, this methodology can be applied to larger clinical dataset in order to detect the disease at the maximum level.
\end{abstract}

Index Terms: Diabetic Retinopathy, Glaucoma, Hemorrhages, lesion, Microaneurysms, Optic Disc.

\section{Introduction}

Glaucoma is an eye disorder that causes an impairment of vision and permanent blindness. Its generally happens for the people over 40 years old, when eye pressure (called intra ocular pressure or IOP) increases, it starts to damage the optic nerve and peripheral vision is lost. This type of disease is problematic free and causes no vision changes at the initial stage later it causes permanent blindness if not treated. It is the foremost reason of blindness worldwide and it is expected to have an effect on over 11 million Indians [1]. India has been estimated to be the second largest place of glaucoma cases by 2020[2]. Glaucoma can be handled with the available treatments, which are more effective if detected early. Regular eye examination automatically burdens the ophthalmologists, since there is limited number of ophthalmologists; there is a vital necessitate for automation in the test practice in order to aware the huge population and mainly for the people living in rural areas to minimize clinical burden. Since the automated computer analysis cannot substitute the clinician, the system aims at detecting fundus images with suspected lesions and at categorizing those by considering severity level of the disease. Then, the interpreted images are sent and reviewed by human expert for review, starting with the suspected most severe cases. Such an automatic screening process can assist to minimize the specialist's complication and assessment time, with the supplementary compensation of objectivity and reproducibility.
Additionally this automatic detection can help to quickly recognize the most severe cases and also helps to concentrate clinical resources on the cases that are more critical and require explicit attention. Diabetes can lead to blood vessel development in the drainage angle of the eye. This can raise eye pressure and cause glaucoma [3]. According to the American Diabetes Association, 40\% of people with diabetes are likely to suffer from glaucoma [4]. The major contribution in the work is to detect the microanuerysms and hemorrhages in the fundus image. Automation can be attained in two levels: first level is segmenting the blood vessels to detect the fundus abnormalities and the second level is classifying the severity level. Certainly, the classification of the severity level, by means of Diabetic Retinopathy (DR) grading, permits more reliable and suitable transfer to treatment centers. The recent research concentrates on the enhancement of an automatic clinical system for computer-assisted screening and grading the level of DR. The fundus images with DR have a part of the eye tissues which would be damaged already is accurately said as red lesion. The retinal red lesions are the major signs of DR and it causes swelling in the retina blood vessels called as microaneurysms [5]. A mass cell accumulation occurs in retina and fluffy patches may also occur in case of bright lesions called hemorrhages. The main objective is to differentiate microaneurysms from stretched out structures to find out early signs of diabetic retinopathy, on the other hand hemorrhages are even more valuable and useful to specify the severity of the disease. 
The foremost step is Pre-processing in which input image is processed for extracting the necessary features; the next step is segmentation where the blood vessels are segmented, then Lesion Identification and finally classifying the severity of the input image.

Some of the problems in the existing system are (i) fails to segment the elongated structures. (ii) It segments only for thick blood vessels and not performing good in thin vessels (iii) pays no attention to useful information from shapes and structures. Figure 1 shows the flow diagram of the proposed system. The input image of the fundus image is acquired from the publicly available dataset (Diaretdb1). The flow of the proposed method is divided into six steps; the primary step involves calibrating a single image against identified values and the applying calibration to uncalibrated image. Second step is pre-processing of the image by giving a flat regular surface to the image known as smoothing and this can also be alone by decomposing image. Then the third step is segmentation, the segmented optic disc of the retina at the point of the entrance of optic nerve, lacking image receptor which creates blindness is also detected to eliminate this portion during the detection of lesions. The fourth step is identifying the people with diabetic retinopathy with corresponding lesions that are identified in the image pre-processing phase. The fifth step comprises of the dynamic shape features extraction. The final step is where the people gets affects with diabetic retinopathy are classified based on their red lesions using Random Forest (RF) classifier.

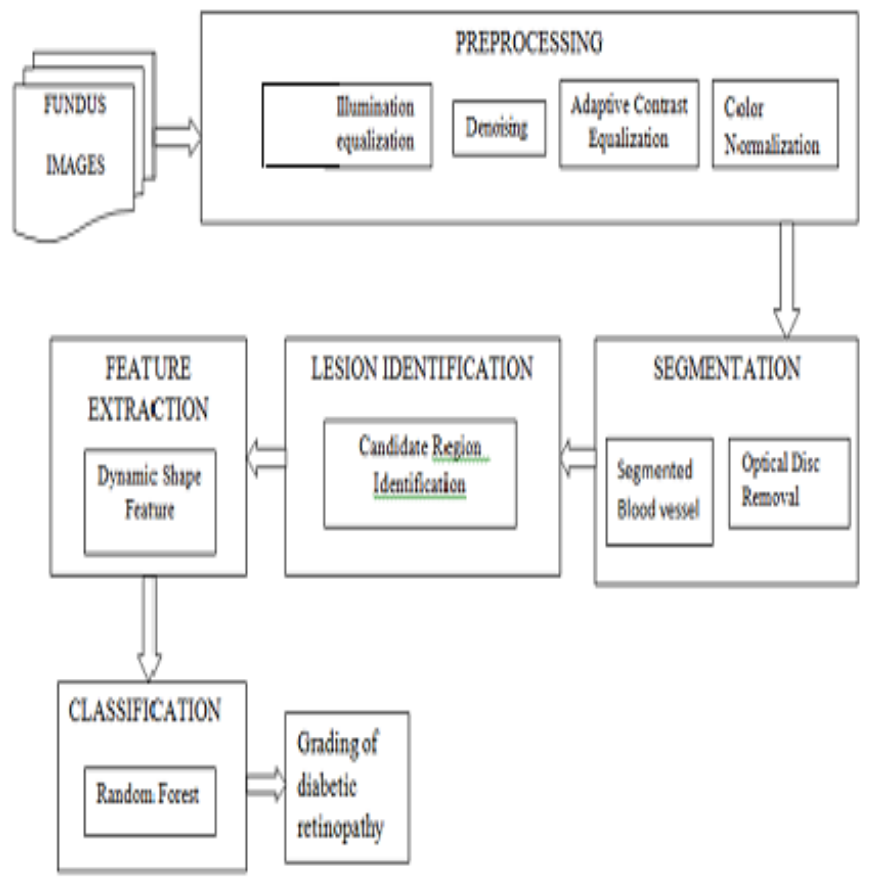

Fig. 1: Flow Diagram of the proposed system

The input image of the fundus is neither increased nor decreased, so the diameter of the spherical portion encircled by the black background i.e. the Region of Interest (ROI) is taken with difference in the size. The DR screening is obtained with a field of view (FOV) at $45^{\circ}$ where the diameter $\mathrm{D}$ is used to set the different size of the filter. The three parameters that are set in this technique are as follows.

1) The average radius of the optical disc is computed as $P 1=\frac{D}{10}$

2) The size of the smallest micro aneurysms is calculated by
$P 2=\frac{D}{360}$

The largest hemorrhages size can be derived using

$P 3=\frac{D}{28}$

\section{Preprocessing}

The illumination of the input fundus image is not uniform in intensity which leads to local luminosity and variation in color contrast. The preprocessing can be done by using the following methods.

\section{A. Illumination equalization}

The illumination equalization technique is used here to overcome the reduction of saturation and brightness of the fundus image. In equalization, the mean filter (M1) of diameter (P1) is applied on every color element which is present in the original fundus image (I) to calculate the illumination of the image. Further the produced colored image is subtracted from the original image and then the intensity of the original image $(\mu)$ is added.

$$
\text { Iie }=I+\mu \cdot I * M I
$$

\section{B. Denoising}

The main aim of an image denoising is to achieve both noise reduction and feature protection. In Denoising (Idn) a mean filter (M2) of diameter (P2) is applied onto the produced image (Iie) of each channel in order to decrease the noise that fallout from the steps which does not involve the smoothing of lesions.

\section{Adaptive Contrast Equalization}

The contrast continuous slow movement from one place to another is approximated by using a local standard deviation (M3) which is used to compute each pixel of its adjacent which is of diameter (D) for different color channel (Ice) places which have low standard deviation indirectly denote that they are areas which have less contrast or which have smooth level background to improve the less contrast area. To enhance the particulars of each portion, the following equation can be used for each color channel. Thus the noise is removed in this phase

$$
I c e=\frac{I d n+1}{\operatorname{Istd}(\operatorname{Idn} *(1-M 3))}
$$

\section{Color Normalization}

This step is necessary to obtain standard color range of the image. The min-max normalization technique is applied to normalize the color, where $\mathrm{x}=(\mathrm{x} 1, \ldots, \mathrm{xn})$ and $\mathrm{zi}$ is the $\mathrm{i}^{\text {th }}$ normalized data.

$$
z i=x i \cdot \min (x) \max (x) \cdot \min (x)
$$

\section{Segmentation}

The optic disc removal is a phase where the false positive in the red lesions need to be removed. In the pre-processed image the entropy based technique is applied to locate the centre of the optic disc. Usually the optic disc is present in the region where there is high 
intensity where the blood vessels possess maximum entropy. The segmented blood vessel task can be created as an energy minimization difficulty in a conditional random field (CRF). In the definition of CRFs, the respective images are verified to graphical representation, where each and every pixel characterizes a node, and all nodes is associated with an edging to their adjacent nodes based on a connectivity rule [6].

\section{Lesion Detection}

Among the candidate region, the several regions correspond to non lesions, such as blood vessels and the left over are the noise in the retinal background. To classify the false positives and true negative lesions, a creative set of elements, the dynamic shape features (DSF's) are based on shape information is used to detect the lesion present in the image.

\section{Feature Extraction}

The blood vessels and lesions which are dark in color and normally these features possess high contrast in the green channel. The red channel and the blue channel are used in the later part for the extraction of certain color features. Micro aneurysms and hemorrhages appear as shapes which contain local minimal intensity especially in green channel [7]. But unfortunately this method is very sensitive to noise. To surmount this drawback the dynamic transformation technique is used. In this technique the minimal regions are rated to their appropriate local contrast. The portions which are noisy and lesions present are usually having low contrast. In diabetic retinopathy several regions correspond to non lesion region such as segments of blood vessels, the flooding level in the topographic representation at each flooding level for each person affected with diabetic retinopathy and catchment basin Bsji.

1) Relative area (Rarea) is calculated by the number of pixels in Bsji, divided by the total number of pixels in the region of interest (ROI).

2) Elongation (Elong) is calculated using the formula: $\boldsymbol{I} \cdot \frac{\boldsymbol{W}}{\boldsymbol{L}}$, where $\mathrm{W}$ is the width and $\mathrm{L}$ is the length of the bounding box of Bsji present along the major axis.

3) Eccentricity (Ecc) is calculated by using: $(L 2-W 2)$,

where $\mathrm{W}$ and $\mathrm{L}$ are the width and length of the bounding box Bsji present along the major axis.

4) Circularity (Circ) is calculated by the ratio of the region of Bsji over it squared perimeter and multiplied by $4 \pi$.

5) Rectangularity (Rect) is calculated by the ratio of the area Bsji over the area of the bounding box along the major axis.

\section{Classification}

The classification phase is the most essential and powerful technique in the recent years since it has number of advantages this technique can be applied to high dimensional and noisy data[8][9]. In our proposed method Random Forest (RF) classifier is used to classify lesions and non-lesions in the fundus image. The Random Forests algorithm is the best algorithm among classification algorithms. This algorithm has the ability to classify enormous amounts of data with greater accuracy of classifying healthy and abnormal images. Random Forests is an collective learning method (also considered as a structure of nearest neighbor predictor) for classification and regression that construct a number of decision trees during the training phase and outputting the category that is the mode of the classes output by individual trees. The algorithmic step of Random forest is shown in Table I.
Table 1. Algorithmic steps of Random Forest

Input: A decision Tree inducer(DT), Iterations (T), Training Sets (S), Sampling portion (R), Number of attributes applied in each decision tree $(\mathrm{N})$

Training set: for $I=1 \ldots \mathrm{T}$

Get a sample $\mathrm{S} 1$ from $\mathrm{S}$ with replacement by means of $\mathrm{R}$

Construct classifier $\mathrm{Ct}$ based on the inducer randomly sample $\mathrm{N}$ of the attributes and Select the finest split.

Classification: new instances classified by classifiers $\mathrm{Ct}(\mathrm{t}=1,2, \ldots \mathrm{T})$, then performed using majority vote.

The international clinical diabetic retinopathy disease severity scale is shown in Table II and this table is used to find the grade of diabetic retinopathy.

Table 2. International clinical diabetic retinopathy disease severity scale [10]

\begin{tabular}{|c|c|}
\hline DR Severity & Finding presence of opthalmoscopy \\
\hline No apparent DR & No Abnormalities \\
\hline Mild NPDR & Microanuerysm only \\
\hline Moderate NPDR & $\begin{array}{l}\text { More than Microanuerysm only but less than } \\
\text { sever NPDR }\end{array}$ \\
\hline Severe NPDR & $\begin{array}{l}\text { Any of the following with no PDR } \\
\text { (i) }>20 \text { intraretinal hemorrhages in each } 4 \\
\text { quadrants } \\
\text { (ii) VB in } 2+\text { quadrants } \\
\text { (iii)Prominent IRMA in } 1+\text { quadrant }\end{array}$ \\
\hline PDR & One or extra NV, PRH,VH \\
\hline
\end{tabular}

NPDR=Non-proliferative Diabetic Retinopathy; PDR=Proliferative Diabetic Retinopathy; NV=Neo vascularization; $\mathrm{PRH}=$ Preretinal Hemorrhage; $\mathrm{VH}=$ Vitreous Hemorrhage; IRMA=Intraretinal Microvascular Abnormalities

\section{Results and Discussions}

The proposed method was tested in the Diaretdb1 dataset for both normal and abnormal images. The result of normal image is shown in fig 2 and the result of abnormal image is shown in figure 3 . The microaneurysm is very less in Figure 2 which implicates that the input fundus image is normal and it is not affected by Glaucoma. From the Figure 3, it is clearly visible that the large number of microaneurysms is found. This indicates that the fundus is affected and the severity is high. In this paper, the performance of segmentation is evaluated in aspects of sensitivity, specificity, and segmentation accuracy. These three terms are most usually related with a Binary classification test and they measure the performance of the test statistically, positive and negative predictive values and positive and negative difference ratios. Sensitivity specifies how well the test predicts one category and Specificity specifies how good the test predicts the other category. Accuracy is likely to measure how good the test predicts together the categories. Let abnormal be the positive for disease and normal be the negative for disease, then,

1) True positive $(\mathrm{TP})=$ number of cases correctly identified as Abnormal.

2) False positive (FP) $=$ number of cases incorrectly identified as Abnormal.

3) True negative $(\mathrm{TN})=$ number of cases correctly identified as Normal.

4) False negative $(\mathrm{FN})=$ the number of cases incorrectly identified as Normal.

The accuracy is to classify the healthy and abnormal cases correctly, i.e., the proportion of $\mathrm{TP}$ and $\mathrm{TN}$ in all of evaluated cases 
Accuracy $=\frac{(T P+T N)}{T P+T N+F P+F N}$

The sensitivity is to determine the abnormal cases correctly, i.e., the proportion of true positive in abnormal cases

Sensitivity $=\frac{T P}{T P+F N}$

The specificity is to determine the normal cases correctly, i.e., the proportion of true negative in normal cases

$$
\text { Specificity }=\frac{T N}{T N+F P}
$$

The performance comparison of segmentation with other methods for sensitivity, specificity and accuracy is shown in Table III [11][12].
Table 3. Performance Comparison

\begin{tabular}{llccc}
\hline \multicolumn{1}{c}{ Dataset } & Algorithm & Se & Sp & Ac \\
\hline Healthy & Proposed & 0.862 & 0.992 & 0.961 \\
Healthy & Frangi et al. & 0.621 & 0.989 & 0.955 \\
Healthy & Odstrcilik et al. & 0.786 & 0.975 & 0.953 \\
Glaucomatous & Proposed & 0.887 & 0.986 & 0.965 \\
Glaucomatous & Frangi et al. & 0.654 & 0.984 & 0.961 \\
Glaucomatous & Odstrcilik et al. & 0.790 & 0.964 & 0.949 \\
DR & Proposed & 0.858 & 0.977 & 0.955 \\
DR & Frangi et al. & 0.590 & 0.972 & 0.946 \\
DR & Odstrcilik et al. & 0.746 & 0.961 & 0.944 \\
\hline
\end{tabular}

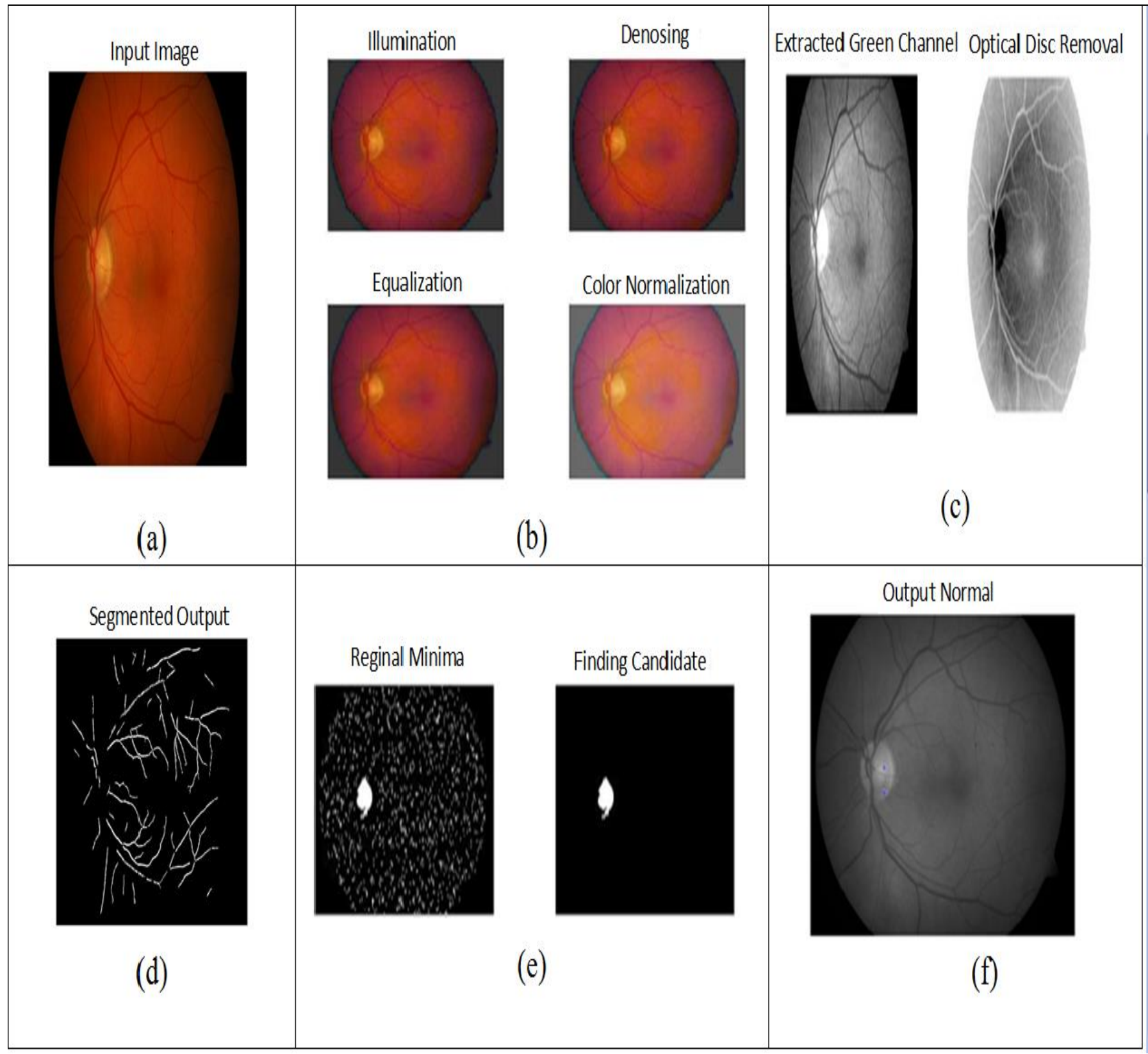

Fig. 2: (a) Input image (b) Preprocessing (c) Optic disc removal (d) Blood vessel segmentation (e) Candidate extraction (f) Classification 


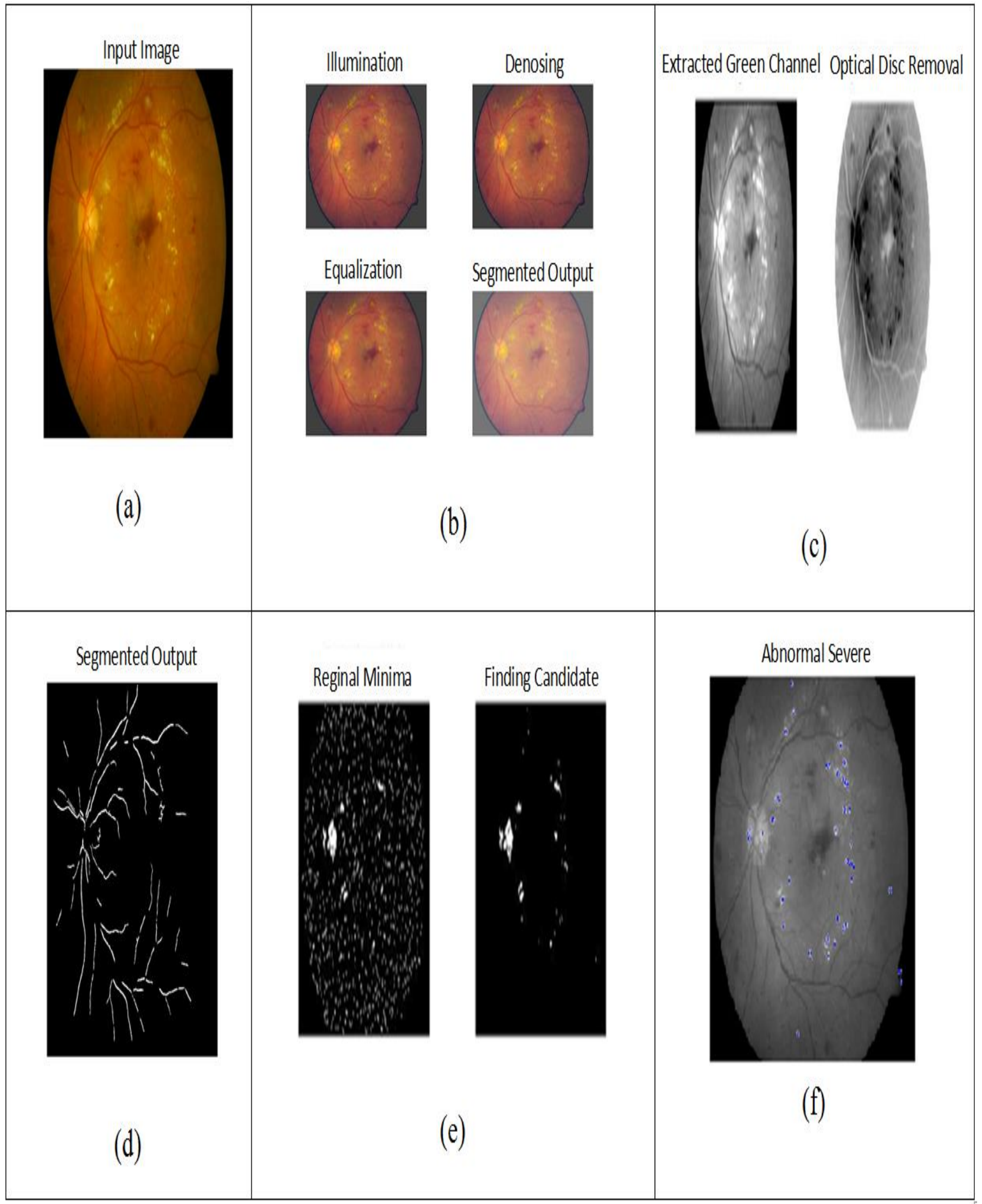

Fig 3. (a) Input image (b) Preprocessing (c) Optic disc removal (d) Blood vessel segmentation (e) Candidate extraction (f) Classification 


\section{Conclusion}

This method has strong performance in detecting both the microaneurysms and hemorrhages in the fundus image for Diaretdb1 dataset with the resolution of $1152 \times 1500$ pixels. This algorithm works very well for diaretdb1 dataset which is related to the diabetic retinopathy. The same can be used for detection of glaucoma with the help of microaneurysms and hemorrhages. In future, this technique can be extended to larger clinical databases and for different image resolution in order to detect the disease and to classify the severity at the maximum level.

\section{References}

[1] Resnikoff S, Pascolini D, Etya'ale D, Kocur I, Pararajasegaram R, Pokharel GP, et al. Global data on visual impairment in the year 2002. Bull World Health Organ 2004; 82: 844-51

[2] George R, Ve RS, Vijaya L. Glaucoma in India: Estimated burden of disease. J Glaucoma 2010; 19:391-7.

[3] R.Shanthi,S.Prabakaran,"Fundus Abnormalities and Image Acquisition Techniques - A Survey", Journal of Chemical and Pharmaceutical Sciences Volume 10, Issue 2 pp 1062-1069, 2017.

[4] https://www.thediabetescouncil.com

[5] Lama Seoud, Thomas Hurtut, JihedChelbi, Farida Cheriet, and J.M.PierreLanglois, "Red Lesion Detection Using Dynamic Shape Features for Diabetic Retinopathy Screening” IEEE Transaction on Medical Imaging, vol.35, no.4, 2016.

[6] Jose Ignacio Orlando, Elena Prokofyeva, and Matthew B. Blaschko, "A Discriminatively Fully Trained Connected Conditional Random Field Model for Blood Vessel Segmentation in Fundus Images", IEEE Transaction on Biomedical Engineering, 2015.

[7] U. T. Nguyen et al., "An effective retinal blood vessel segmentation method using multi-scale line detection," ELSEVIER on pattern Recognition, vol. 46, no. 3pp.703-715, 2013.

[8] Joao V. B. Soares, Jorge J. G. Leandro, Herbert F. Jelinek, Roberto M. Cesar Jr Michael J. Cree, "Retinal Vessel Segmentation Using the 2-D Gabor Wavelet and Supervised Classification", IEEE Transaction on Medical Imaging, vol. 25, no. 9, 2006

[9] Yi Yuan, Xiangyun $\mathrm{Hu}$, "Random Forest And Objected-Based Classification For Forest Pest Extraction From Uav Aerial Imagery", The International Archives of the Photogrammetry, Remote Sensing and Spatial Information Sciences, Volume XLI-B1, 2016 XXIII ISPRS Congress, 12-19 July 2016,

[10] Wikinson cp et al opthalmology 2003;1 10:1677-1682. American Academy of Ophthalmology Retina Panel, Preferred Practice Patterns Committee. Diabetic retinopathy. San Francisco (CA): American Academy of Ophthalmology (AAO); 2003.

[11] Frangi et al.,"Multiscale vessel enhancement filtering", In medical Image Computing and computer-assisted intervention volume 1496 springer verlag pp 130-137 1998.

[12] J. Odstrcilik et al., "Retinal vessel segmentation by improved matched filtering: Evaluation on a new high-resolution fundus image database, "IET Image Process., vol. 7, no. 4, pp. 373-383, 2013.

[13] T. Padmapriya and V. Saminadan, "Improving Throughput for Downlink Multi user MIMO-LTE Advanced Networks using SINR approximation and Hierarchical CSI feedback", International Journal of Mobile Design Network and Innovation- Inderscience Publisher, ISSN : 1744-2850 vol. 6, no.1, pp. 14-23, May 2015.

[14] S.V.Manikanthan and K.srividhya "An Android based secure access control using ARM and cloud computing", Published in: Electronics and Communication Systems (ICECS), 2015 2nd International Conference on 26-27 Feb.2015, Publisher:IEEE, DOI:10.1109/ECS.2015.7124833. 